\title{
Urethral Emulsion Dosage Form
}

National Cancer Institute

\section{Source}

National Cancer Institute. Urethral Emulsion Dosage Form. NCI Thesaurus. Code C150005.

Liquid preparation consisting of an emulsion intended for urethral use by means of a suitable applicator. 